\title{
A HOLISTIC MEASUREMENT MODEL OF MOVEMENT COMPETENCY IN
}

\section{CHILDREN}

Rudd, J. ${ }^{1}$, Butson, M, L. ${ }^{1}$, Barnett, L. ${ }^{2}$, Farrow, D. ${ }^{1,3}$, Berry, J. ${ }^{1}$, Borkoles, E. ${ }^{1}$, \& Polman, $\mathrm{R}^{1,4}$

1. Institute of Sport Exercise and Active Living, Victoria University, Melbourne, Australia 2. School of Health and Social Development, Deakin University, Melbourne, Australia 3. Movement Science, Australian Institute of Sport, Canberra, Australia 4. Psychology Department, Bournemouth University, United Kingdom

Address of correspondence: James Rudd, Institute of Sport, Exercise and Active Living, College of Sport and Exercise Science, Victoria University, Melbourne, Australia. Telephone +61 3 99199574; email: james.rudd@vu.edu.au 


\begin{abstract}
Different countries have different methods for assessing movement competence in children, however it is unclear whether the test batteries that are used measure the same aspects of movement competence. The aim of this paper was to 1) Investigate whether the Test of Gross Motor Development (TGMD-2) and Körperkoordinations Test für Kinder (KTK) measure the same aspects of children's movement competence and 2.) Examine the factorial structure of the TGMD-2 and KTK in a sample of Australian children. A total of 158 children participated (M age $=9.5$; SD 2.2). First, confirmatory factor analysis examined the independent factorial structure of the KTK and TGMD-2. Second, it was investigated whether locomotor, object control and body coordination loaded on the latent variable Movement Competency. Confirmatory factor analysis indicated an adequate fit for both the KTK and TGMD-2. An adequate fit was also achieved for the final model. In this model locomotor $(\mathrm{r}=.86)$, object control $(\mathrm{r}=.71)$ and body coordination $(\mathrm{r}=.52)$ loaded on movement competence. Findings support our hypothesis that the TGMD-2 and KTK measure discrete aspects of movement competence. Future researchers and practitioners should consider using a wider range of test batteries to assess movement competence.
\end{abstract}

Key Words: Movement Competence, Fundamental Movement Skills, Body Coordination 


\section{INTRODUCTION}

Movement competency is an integral component of physical literacy, which has been defined as having the movement competence, knowledge, skills and attitudes to live a healthy life and also is an advocate for others to do the same (Whitehead, 2007). Having movement skill competence is important as it has been shown to be an important predictor of regular physical activity and health related fitness in children (Cattuzzo et al., 2014; Lubans, Morgan, Cliff, Barnett, \& Okely, 2010). It is suggested that movement competency is a fundamental aspect of childhood development with a lasting influence on aspects of health across the lifespan (Ahnert, Schneider, \& Bös, 2009; Robinson et al., in press ; Stodden et al., 2008)

A limitation in the current definition of physical literacy is ambiguity about what constitutes movement competence. However, this has not stopped physical literacy becoming an important focus of physical education curricula (Mandigo, Francis, Lodewyk, \& Lopez, 2009) and in the promotion of physical activity (Whitehead, 2001). For example, the physical education curricula of Australia (ACARA, 2011) and England (Department of Education, 2013) aim to promote lifelong participation in physical activity through the development of physical literacy, with a focus on developing movement competence in children and through the development of self and social awareness, self-regulation and responsible decision making, to foster overall personal well-being. The result being a physically educated person with the ability to use these skills in everyday life and developing a disposition towards purposeful physical activity being an integral part of daily living (Castelli, Centeio, Beighle, Carson, \& Nicksic, 2014). However, in the effort to create physically literate children it is important that the concept of movement competency is better understood and defined.

Gallahue, Ozmun, \& Goodway, (2012) classify movement competence within three distinct holistic categories: locomotion, object control, and stability skills and state that there are typical developmental progressions between skills and also between the categories. They 
surmise that children need to master certain stability skills before they can progress onto locomotor skills and that children seem to form rudimentary stability and locomotor skills earlier than they develop object control skills (Gallahue et al., 2012). According to Whitehead, movement competency is multi-dimensional in nature, containing three interrelated constructs: simple movement capacities, combined movement capacities and complex movement capacities (Whitehead, 2010). Such a multi-dimensional conceptualisation of movement competence is common in the human movement literature. Dynamical Systems Theory and cognitive psychology both provide a multi-dimensional taxonomy of movement skills to describe movement competence, though they do offer differing hypotheses of how movement competency is developed (Burton, Miller, \& Miller, 1998; Fleishman, 1975).

Overall, there is still a lack of consensus about what movement competence encompasses. An important reason for this disagreement is the variation in measurement methods (Giblin, Collins, \& Button, 2014). For example, in North America the Test of Gross Motor Development (TGMD) (Ulrich, 2000) has been a test battery of choice to examine children's movement competency. The TGMD is a process oriented test battery that measures competency in a set of motor skills deemed essential for predicting participation in PA and sport. The motor skills are known as fundamental movement skills (FMS) and have been subdivided into two categories called locomotor and object control skills. Confirmatory factor analysis on an American sample has provided evidence for the proposed hierarchical structure of the TGMD-2, suggesting that the TGMD-2 provides a good evaluation of children's gross motor competency (Ulrich, 2000).

The Körperkoordinations Test für Kinder (KTK) has been developed in Germany to examine non-sport specific gross body coordination in children. The KTK has been shown to have good reliability (test-retest reliability between .80 and .96) and factorial structure, where 
adequate predictive validity has been shown by its ability to distinguish between brain damaged and normal children (Kiphard \& Schilling, 2007; Kiphard \& Schilling, 1974).

There is a growing body of evidence that assessment tools should not be used interchangeably, Fransen, et al (2014) compared the KTK and Bruininks-Oseretsky Test of Motor Proficiency (BOT-2; Bruininks \& Bruininks, 2005) in primary school children and found only a moderate association between the two tests. These findings are similar to other convergent validity studies (Smits-Engelsman, Henderson, and Michels 1998; Logan, Robinson, and Getchell 2011). It is currently unclear whether the TGMD-2 and KTK are measuring the same or different aspects of children's movement competency. If the two test batteries measure different aspects of movement competence, this would suggest key information on a child's movement competency could be missed if only one test battery is used. So the first aim was to explore whether the two test batteries measure different aspects of movement competence. We hypothesise that movement competence includes both locomotor and object control competence and that this is distinct from body coordination. To date, no Australian studies have examined the factorial structure of the TGMD-2. Similarly, no studies examining the KTK, outside of Europe, have reported whether their proposed factorial structure is invariant across samples of different cultural backgrounds. A secondary aim of the present research was therefore to examine the factorial structure of both the TGMD-2 and KTK in a sample of Australian children.

\section{METHOD}

\section{Participants}

In total, 158 children aged 6-12 participated in the study ( $M$ age $=9.5$ SD 2.2$), 86$ (54\%) were boys and 72 (46\%) were girls. The study was approved by the University Ethics Committee and Victoria Department of Education and Early Childhood Development, and parental consent was obtained for all participants. 


\section{Test Battery}

The Test of Gross Motor Development-2 (TGMD-2) (Ulrich, 2000) assesses proficiency in six locomotor skills (run, hop, slide, gallop, leap, horizontal jump) and six object control skills (striking a stationary ball, stationary dribble, catch, kick, overhand throw, underhand roll). Each participant completes all 12 skills of the TGMD-2 and is given one practice attempt and two assessment trials for each skill. For each skill, skill components are marked as 'present' or 'absent'.

The Körperkoordinations Test für Kinder (KTK) (Kiphard \& Schilling, 2007) is an outcome based assessment that consists of four non-sport specific sub-tests that measure gross motor coordination. Reverse balancing requires participants to walk backwards along three different balance beams, with increasing levels of difficulty due to the width of the beams decreasing from $6 \mathrm{~cm}$ to $4.5 \mathrm{~cm}$ to $3 \mathrm{~cm}$ respectively. Moving platforms requires participants to move laterally for 20 seconds across the floor using two wooden platforms. Participants step from one platform to the next platform, and then move the first platform to their side in the direction they are travelling and step on to it. Hopping for height requires participants to hop on one leg over an increasing number of $5 \mathrm{~cm}$ foam blocks to a maximum of 12 blocks. Participants have to begin hopping $1.5 \mathrm{~m}$ away from the foam blocks, hop up to and over the foam block and complete a further two hops for the trial to be deemed successful. The final task is continuous lateral jumping in which participants are required to complete as many sideways jumps as they can, with feet together, over a wooden slat in 15 seconds. 


\section{Training and Reliability}

A total of 10 Research Assistants (RAs) each received six hours training in the administration of the TGMD-2 and KTK. At the end of this training period the RAs administering the KTK assessment tool scores were compared and achieved 94\% agreement reliability. Two of the RAs received an additional three hours training on coding each of the 12 TGMD-2 skills.

These two RAs independently coded videos of 15 children who completed the 12 TGMD-2 skills. To determine the agreement between the two RAs total scores for each subset (locomotive and object control) were first z-transformed. Next limits of agreement for each subset were calculated based on the mean difference between the two assessor's scores and the respective standard deviation of these differences (Bland \& Altman, 1986; Nevill, 1996). The 95\% limit above and below the mean for locomotor skills were -0.7 to 0.7 and for object control skills $95 \%$ limit agreements were -0.6 to 0.6 . The RA's $95 \%$ confidence intervals are within one standard deviation (1.96) and contains zero, demonstrating that the two RA's have excellent inter-rater reliability.

\section{Procedure}

The assessments of TGMD-2 and KTK were carried out in a large sports hall. Groups of four participants rotated around five stations, each manned by two trained RA's, and the TGMD-2 stations were video recorded for subsequent coding. The four KTK assessments were divided into two stations whereas the TGMD-2 was split into object control and locomotive skills.

\section{Statistical Analysis}

Raw scores for each TGMD-2 skill and the four KTK tests were transformed onto the same scale through z-transformation. Following this, data was assessed for violation of the assumptions of normality and for outliers. 
Confirmatory factor analysis was used to examine the factorial structure of the KTK and TGMD-2 using AMOS 22. First, a confirmatory factor analysis was conducted to examine whether the individual tests of the KTK served as a good indicator for the latent factor Body Coordination. Following this, two confirmatory factor analyses were conducted to assess the fit of the TGMD-2 skills into locomotor and object control latent factors respectively. In the instance of an adequate fit, a fourth confirmatory factor analysis was conducted to examine the hierarchical nature of the TGMD-2 by testing whether locomotor and object control loaded on the higher order variable, FMS. If the fit was found to be inadequate, the model was respecified. Finally, if the fit was adequate, it was examined whether the empirical data fitted the hypothesised model in which both FMS and body coordination loaded on the latent variable Movement Competency.

\section{Goodness of Fit}

Confirmatory factor analysis was conducted with the maximum likelihood method of estimation. In order to specify a model containing latent variables for all factors, error variance was set at zero. Residuals from the observed variables were allowed to co-vary within each specified factor, as indicated by corresponding arrows in path diagrams. Several goodness of fit measures were used to describe the models. In addition to the Chi square $(\chi 2)$ statistic, which is influenced by sample size (Ullman, 2006), the following fit indices were considered: Chi square/DF ( $\left.\chi^{2} / \mathrm{DF}\right)$; Comparative fit index (CFI) (Bentler, 1990); Root mean square error of approximation (RMSEA) (Browne, Cudeck, \& Bollen, 1993); Standardised root mean residual (SRMR) (Bollen, 1989); and the P of close fit PCLOSE (Hu \& Bentler, 1999).

The $\chi^{2}$ statistic is a measure of overall fit of the model to the data, with a nonsignificant $\mathrm{P}$-value $(\mathrm{P}>.05)$ indicating a good fit. Also, $\chi^{2}$ divided by the degrees of freedom $\left(\chi^{2} / \mathrm{df}\right)$ provides an indicator of fit with values of $<2$ considered adequate fit. Comparative fit 
index values of .90 or above indicate an adequate fit. Root mean square error of approximation values of .06 or lower and standardised root mean residuals values of .08 or lower indicate a close fit when these statistics are taken together (Kline, 2011). However, it should be noted that Vandenbergh and Lance (2000) have suggested that cut-off values of .08 for root mean square error of approximation and .10 for standardised root mean residuals are acceptable lower bounds of good model fit (Vandenberg \& Lance, 2000). Finally, the PCLOSE should be non-significant $(\mathrm{P}>$.05) (Browne \& Cudeck, 1992; Hooper, Coughlan, \& Mullen, 2008).

\section{RESULTS}

The Mardia (1970) test for multivariate kurtosis was undertaken (Mardia, 1970), following Kline's (2011) suggestion that critical ratio of > 3 are of a concern (Kline, 2011). None of the models showed problematic levels of skewness or kurtosis. Mean scores and standard deviations are reported below for all children on both test batteries.

Table 1 near here

\section{Confirmatory Factor Analysis for the KTK}

The confirmatory factor analysis for the KTK provided an adequate model fit $\left(\chi^{2}(2 \mathrm{df})\right.$ $\left.=1.49 ; \mathrm{P}=.47 ; \chi^{2} / \mathrm{df}=0.75 ; \mathrm{CFI}=1.00 ; \mathrm{SRMR}=.01 ; \mathrm{RMSEA}=.01 ; \mathrm{PCLOSE}=.60\right)$. All four observed measures had a strong effect on the latent variable Body Coordination (see Figure 1)

\section{Confirmatory Factor Analysis of the TGMD-2}

The Confirmatory factor analysis for locomotive skills showed an adequate fit for the overall model $\left(\chi^{2}(9 \mathrm{df})=9.21 ; \mathrm{P}=.42 ; \chi^{2} / \mathrm{df}=1.02 ; \mathrm{CFI}=.99 ; \mathrm{SRMR}=.05 ; \mathrm{RMSEA}=.01\right.$; PCLOSE $=.69$ ). The initial confirmatory factor analysis for object control provided an inadequate fit $\left(\chi^{2}(9)=27.54 ; \chi^{2} / \mathrm{df}=1.34 ; \mathrm{P}=.001 ; \mathrm{CFI}=.80 ; \mathrm{SRMR}=.07 ; \mathrm{RMSEA}=.11\right.$; PCLOSE $=.02$ ). The modification indices indicated that the error term for the observed 
variable Throw was related to the error term of the observed variable Strike. As such, the error terms for these variables were co-varied. The revised model for object control provided an adequate fit $\left(\chi^{2}(8)=10.13, \mathrm{P}=.26 ; \chi^{2} / \mathrm{df}=1.26 ; \mathrm{CFI}=.98 ; \mathrm{SRMR}=.04 ; \mathrm{RMSEA}=.04\right.$; PCLOSE $=.52)$.

FMS hierarchical model for the TGMD-2 (see Figure 2 ) showed an adequate fit $\left(\chi^{2}\right.$ $\left.(52)=71.07 ; \mathrm{P}=.04 ; \chi^{2} / \mathrm{df}=1.36 ; \mathrm{CFI}=.86 ; \mathrm{SRMR}=.07 ; \mathrm{RMSEA}=.05 ; \mathrm{PCLOSE}=.52\right)$. In this model object control had more effect $(r=.67)$ than locomotor $(r=.39)$ on overall fundamental movement skill. The catch was found to load very weakly onto object control (r $=.08$ ) though it did still contribute to the overall model fit (see Figure 2).

\section{Movement Competency Structural Model}

The initial confirmatory factor analysis for the hypothesised movement competency model (see Figure 3) showed an improper solution caused by over specification of the TGMD-2 skills with two second order factors (locomotor and object control) and the higher order factor FMS both explaining the TGMD-2 skills; therefore creating an unstable fit. A second confirmatory factor analysis for movement competence was carried out (see Figure 4). The FMS latent variable was dropped from the movement competency model to avoid over specification of the TGMD-2 skills. The three second order latent variables:

coordination, object control and locomotor now loaded directly into movement competency. An adequate fit was achieved $\left(\chi^{2}(102)=155.40 ; \mathrm{P}=.001 ; \chi^{2} / \mathrm{df}=1.52 ; \mathrm{CFI}=.89 ; \mathrm{SRMR}=\right.$ $.09 ;$ RMSEA = .06; PCLOSE $=.24)$. In this model locomotor $(\mathrm{r}=.86)$, object control $(\mathrm{r}=$ $.71)$ and body coordination $(\mathrm{r}=.52)$ loaded on movement competence. The catch also now provided a higher loading on object control.

\section{DISCUSSION}

This study examined the relationship between the TGMD-2 and the KTK and tested its factorial structure in a sample of Australian children. Both the TGMD-2 and KTK, when 
examined independently, showed good model fit in our sample. In addition, findings support our hypothesis that the TGMD-2 and KTK measure discrete aspects of the movement competency construct.

The proposed movement competency model in this study suggests that both object control and locomotor skills of the TGMD-2 and the body coordination skills of the KTK are related to the overall concept of movement competency. The final model provided an adequate fit and there did not appear to be any redundancies. An important implication of this finding is that, if used individually, these commonly used assessment batteries provide only a limited view of the overall movement competence of children. To obtain a more holistic picture of the movement competencies of children future research should examine both FMS and body coordination skills.

The KTK is a product assessment test battery with each skill outcome being assessed quantitatively (i.e., number of jumps completed in a specific time). In contrast the TGMD-2 provides a qualitative assessment of skill execution (i.e., whether a child does or does not demonstrate specific component). Although the TGMD-2 does not measure the outcome of a given movement sequence, it is implicitly assumed that the underlying process is associated with successful outcomes. Indeed, empirical evidence suggests strong associations between skill process and skill outcomes. Miller (2007) investigated the correlation between process and product scores of a two-handed sidearm strike in children. A significant relationship was found between the product and process scores for each trial (correlations ranging from $r=.51$ to .66) demonstrating a consistent association between technique and outcome (Miller, Vine, \& Larkin, 2007). Roberton and Konczak (2001) compared the product and process of the overarm throw and reported a significant correlation between quantitative (ball velocity) and quality of performance in primary school children (Roberton \& Konczak, 2001). Both these 
studies provide evidence for a positive relationship between process and product FMS measures.

The separation of product and process measurement of movement competence has been questioned (Stodden et al., 2008). The choice of a process or product test battery, in this respect, might be indicative of theoretical beliefs on how movement competence is formulated. For example, in general terms an ecological dynamics theorists may favour a process orientated approach whereas a cognitive psychologist may adopt a product approach. Our analysis suggests that both assessment strategies provide a useful assessment of movement competence and that both strategies should be used concurrently to obtain a more holistic assessment of the movement competence of children.

Two recent systematic reviews and meta-analyses have provided evidence that FMS interventions can be successful in motor skill development in children (Logan, Robinson, Wilson, \& Lucas, 2012; Morgan et al., 2013). These interventions only focused on aspects of FMS development rather than development of FMS and body coordination. The results of the present study and work by Erikson (2008) suggest that children's movement competency encompasses a number of additional components besides FMS and that interventions based solely on the development of FMS might not provide adequate development of body coordination resulting in a lack overall movement competence in the long-term.

Our proposed movement competence model suggests that for children to be truly competent they should participate in a wide range of activities. This is supported by evidence demonstrating that elite athletes do not specialise in their specific sport from an early age but participate in a wide range of activities throughout childhood and specialise when they are older (Côté \& Fraser-Thomas, 2007). To this extent, children should be encouraged and given the opportunity by parents, schools and clubs to take part in task oriented body coordination movement activities which focus on moving and controlling the body in gravity defying ways 
to encourage the development of movement fluency, rhythm, timing and body strength. Suitable examples of such activities would be gymnastics, dance and martial arts. Activities such as these should be experienced alongside learning key object control and locomotive skills, learnt through deliberate play (Côté \& Fraser-Thomas, 2008) and traditional sports. Together they will promote a strong foundation in overall movement competence.

Our results highlight that movement competence is a multi-dimensional concept and may not be recorded adequately by one test battery. As such, this model may still fail to capture all aspects of children's movement competence. In turn this results in current interventions typically only being designed to address select aspects of movement competence. In addition, the movement competence model presented in the present study needs to be tested in larger samples of children across different countries to demonstrate its generalizability.

In conclusion, the results of the present study provide support for the factorial structure of the TGMD-2 and KTK in a sample of Australian children. In addition, movement competence consist of both FMS (process) and body coordination (product) activities. As such this study suggests that future studies and interventions should consider using testing batteries which provide a more holistic way of assessing movement competency in children. 


\section{REFERENCES}

Ahnert, J., Schneider, W., \& Bös, K. (2009). Developmental changes and individual stability of motor abilities from the preschool period to young adulthood. In: W, Schneider, M, Bullock (Eds.). Human Development from Early Childhood to Early Adulthood: Evidence from the Munich Longitudinal Study on the Genesis of Individual Competencies (LOGIC) (pp 35-62). Mahwah, NJ: Erlbaum

ACARA. (2011). Draft Shape of the Australian Curriculum: Health and Physical Education. Australian Curriculum Assessment and Reporting Authority. Sydney: ACARA.

Barnett, L.M., Van Beurden, E., Morgan, P.J., Brooks, L.O., \& Beard J.R. (2009). Childhood motor skill proficiency as a predictor of adolescent physical activity. Journal of Adolescent Health, 44: 252-259.

Bland, M. J., \& Altman, D. G. (1986). Statistical methods for assessing agreement between two methods of clinical measurement. The Lancet, 327(8476), 307-310. http://doi.org/10.1016/S0140-6736(86)90837-8

Bentler, P.M. (1990). Comparative fit indexes in structural models. Psychological Bulletin, 107(2), 238-246.

Bollen, KA. (1989). A new incremental fit index for general structural equation models. Sociological Methods \& Research, 17(3), 303-316.

Bruininks, R. (2005). Bruininks B. Bruininks-Oseretsky Test of Motor Proficiency Second Edition (BOT2). Circle Pines, MN: AGS Publishing.

Browne, M.W., \& Cudeck, R., (1992). Alternative ways of assessing model fit. Sociological Methods \& Research, 21(2), 230-258. 
Browne, M.W., Cudeck, R., \& Bollen, K.A. (1993). Alternative ways of assessing model fit. Sage Focus Editions, 154, 136.

Burton, A.W., Miller, D.E., \& Miller. D. (1998). Movement skill assessment: Champaign, IL: Human Kinetics.

Castelli, D.M., Centeio, E.E., Beighle, A.E., Carson, R.L., \& Nicksic, H.M. (2014). Physical literacy and Comprehensive School Physical Activity Programs. Preventive medicine; 66: 95100.

Cattuzzo, M. T., Dos Santos Henrique, R., Ré, A. H., de Oliveira, I. S., Melo, B. M., de Sousa M... \& Stodden, D. (2014). Motor competence and health related physical fitness in youth: A systematic review. Journal of Science and Medicine in Sport. doi: http://doi.org/10.1016/j.jsams.2014.12.004

Côté, J., \& Fraser-Thomas, J. (2007). Youth involvement in sport. in: P, Crocker, (Eds.) Sport psychology: A Canadian perspective, 270-298.

Côté, J, \& Fraser-Thomas, J. (2008). Play, practice, and athlete development. In D. Farrow, J. Baker, C. MacMahon (Eds.) Developing elite Sport Performance: Lessons from theory and practice. 17-24. New York: Routledge.

Department of Education, (2013). National curriculum in England: physical education programmes of study. (dfe-00176-2013). England: GOV.UK.

Ericsson, I. (2008). To measure and improve motor skills in practice. International Journal of Pediatric Obesity, 3, 21-27.

Fleishman, E.A. (1975). Toward a taxonomy of human performance. American Psychologist. 30 (12), $1127-1149$. 
Gallahue, D. L., Ozmun, J. C., \& Goodway, J. (2012). Understanding Motor Development: Infants, Children, Adolescents, Adults. McGraw-Hill.

Fransen, J., D’Hondt, E., Bourgois, J., Vaeyens, R., Philippaerts, R. M., \& Lenoir, M. (2014). Motor competence assessment in children: Convergent and discriminant validity between the BOT-2 Short Form and KTK testing batteries. Research in developmental disabilities, 35(6), $1375-1383$.

Giblin, S., Collins, D., \& Button, C. (2014) Physical Literacy: Importance, Assessment and Future Directions. Sports medicine. 44 (9) 1177-1184.

Hooper, D., Coughlan, J., \& Mullen, M. (2008) Structural equation modelling: guidelines for determining model fit. Journal of Business Research Methods, 6(1), 53-60.

Hu, Li-tze, \& Bentler, P.M. (1999) Cut-off criteria for fit indexes in covariance structure analysis: Conventional criteria versus new alternatives. Structural Equation Modeling: A Multidisciplinary Journal. 6 (1) 1-55.

Kiphard, E.J., \& Schilling, F. (2007). Körperkoordinationstest für Kinder. 2. Überarbeitete undergänzte Auflage. Weinheim: Beltz Test GmbH

Kiphard, E.J. \& Schilling, F. (1974). Körperkoordinationstest für Kinder. Weinheim: Beltz Test GmbH

Kline, R.B. (2011). Principles and practice of structural equation modelling: Guilford, UK: Guilford press.

Logan, S., Robinson, L., Wilson, A., \& Lucas, W. (2012). Getting the fundamentals of movement: a meta-analysis of the effectiveness of motor skill interventions in children. Child: care, health and development. 38. 305-315. 
Logan, S. W., Robinson, L. E., \& Getchell, N. (2011). The comparison of performances of preschool children on two motor assessments. Perceptual and motor skills, 113(3), 715-723.

Lubans, D.R., Morgan, P.J., Cliff, D.P., Barnett, L.M., \& Okely, A.D. (2010). Fundamental movement skills in children and adolescents. Sports medicine. 40. (12) 1019-1035.

Mandigo, J., Francis, N., Lodewyk, K., \& Lopez, R. (2009) Physical literacy for educators. Physical Activity Health Education Journal. 75. 27-30.

Mardia, K.V. (1970) Measures of multivariate skewness and kurtosis with applications. Biometrika. 57 (3), 519-530.

Miller, J., Vine. K., \& Larkin, D.(2007) The relationship of process and product performance of the two-handed sidearm strike. Physical Education and Sport Pedagogy. 12, (1) 61-76.

Morgan. P.J., Barnett. L.M., Cliff. D.P., Okely. A.D., Scott. H.A., Cohen. K.E., \& Lubans. D.R,. (2013) Fundamental movement skill interventions in youth: A systematic review and meta-analysis. Pediatrics. 132 (5) 13161- 1383.

Nevill, A. (1996) Validity and measurement agreement in sports performance. Journal Of Sports Sciences. 14(3):199.

Nunnally, J. C., \& Bernstein, I. H. (1994). Psychometric theory, 752 ( ${ }^{\text {rd }}$ ed.) New York: McGraw-Hill.

Roberton, M.A., \& Konczak, J. (2001) Predicting children's overarm throw ball velocities from their developmental levels in throwing. Research Quarterly for Exercise and Sport. 72. (2) 91-103. 
Robinson, L. E., Stodden, D. F., Barnett, M., L., Lopes, V. P., Logan, W., S., Rodrigues, P. L., \& D'Hondt, E. (in press). Motor Competence and its Effect on Positive Developmental Trajectories of Health. Sports Medicine.

Smits-Engelsman, B. C., Henderson, S. E., \& Michels, C. G. (1998). The assessment of children with Developmental Coordination Disorders in the Netherlands: The relationship between the Movement Assessment Battery for Children and the Körperkoordinations Test für Kinder. Human Movement Science, 17(4), 699-709.

Stodden, D.F., Goodway, J.D., Langendorfer, S.J., Roberton, M.A., Rudisill, M.E., Garcia C., \& Garcia L.E. (2008) A developmental perspective on the role of motor skill competence in physical activity: An emergent relationship. Quest. 60. 290-306.

Ullman, J.B. (2006). Structural equation modeling: Reviewing the basics and moving forward. Journal of Personality Assessment. 87 (1) 35-50.

Ulrich, DA. (2000) Test of gross motor development-2. Austin: Prod-Ed.

Vandenberg, R.J., Lance, C.E. (2000). A review and synthesis of the measurement invariance literature: Suggestions, practices, and recommendations for organizational research. Organizational research methods. 3, (1) 4-70. doi: 10.1177/109442810031002

Whitehead, M. The concept of physical literacy. (2001) European Journal of Physical Education. 6, (2) 127-138.

Whitehead, M. (2007) Physical literacy: Philosophical considerations in relation to developing a sense of self, universality and propositional knowledge. Sports Ethics and Philosophy. 1. 281-298.

Whitehead, M. (2010) Physical literacy: Throughout the lifecourse: London: Routledge. 
Figure 1: Confirmatory Factor Analysis for the Körperkoordinations Test für Kinder Figure 2: Fundamental Movement Skill hierarchical model for the Test of Gross Motor Development 2

Figure 3: Movement competency model Figure 4: Final model of Movement Competence 